\title{
Vascular findings in CTA body and extremity of critically ill COVID-19 patients: commonly encountered vascular complications with review of literature
}

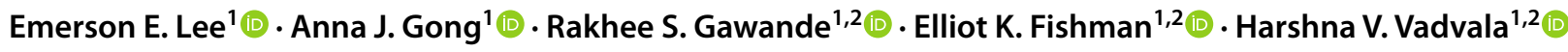

Received: 29 November 2021 / Accepted: 21 December 2021 / Published online: 22 January 2022

○) American Society of Emergency Radiology 2021

\begin{abstract}
The purpose of our review is to discuss the role of CT angiography (CTA) in evaluating a variety of vascular complications in critically ill COVID-19 patients. The COVID-19 pandemic continues to be a worldwide health threat. While COVID-19 pneumonia is the most common and well-recognized presentation of COVID-19, severely ill hospitalized patients often present with extrapulmonary systemic findings. Vascular complications occur not only due to known viral-induced vasculopathy, coagulopathy, and related "cytokine storm," but also due to anticoagulation medication used during hospitalization. There is a paucity of articles describing extrapulmonary vascular findings, especially in critically ill COVID-19 patients. In our article, we discuss commonly encountered vascular imaging findings in the body (chest, abdomen, and pelvis) and extremities, the importance of early radiological detection, and the role of CTA in the management of critically ill COVID-19 patients.
\end{abstract}

Keywords CT angiography of body and extremity $\cdot$ COVID-19 V Vascular findings · Critically ill patients

\section{Introduction}

The COVID-19 pandemic caused by severe acute respiratory syndrome coronavirus-2 (SARS-CoV-2) has been associated with significant morbidity and mortality, primarily due to

Emerson E. Lee and Anna J. Gong contributed equally.

Harshna V. Vadvala

drharshna@gmail.com; hvadval1@jhmi.edu

Emerson E. Lee

elee162@jhmi.edu

Anna J. Gong

agong4@jh.edu

Rakhee S. Gawande

rgawand1@jhmi.edu

Elliot K. Fishman

efishman@jhmi.edu

1 Johns Hopkins Hospital, Johns Hopkins University School of Medicine, Johns Hopkins University, 601 North Caroline Street, Baltimore, MD 21287, USA

2 Department of Radiology and Radiological Science, Johns Hopkins Medicine, Johns Hopkins Hospital, Johns Hopkins University, 601 North Caroline Street, Baltimore, MD 21287, USA respiratory failure and subsequent multisystem involvement. Early radiographic investigations focused on lung findings, but COVID-19 infection is increasingly recognized as a multi-organ disease. Notably, COVID-19 infection is further complicated by coagulopathy, with disseminated intravascular coagulation (DIC) reported in a majority of patients who die of COVID infection [1]. Although thromboembolic events in critically ill COVID patients are well-recognized, anticoagulation strategy remains complex: while the incidence of thromboembolic events is considerable, especially in ICU patients, the occurrence of potentially lethal hemorrhagic events and overall inpatient mortality increases with therapeutic-dose anticoagulation [2]. Thus, escalation of anticoagulation dose to therapeutic levels requires careful consideration of risk factors and therapeutic benefit.

Most patients who test positive for COVID-19 demonstrate a hypercoagulable state, including elevated D-dimer, troponins, ferritin levels, and leukocytosis; however, baseline laboratory features are poorly predictive of which patients will experience thromboembolic events [3]. Thus, imaging plays an active role in appropriate diagnosis and management of these critically ill patients, including initiation of therapeutic-dose anticoagulation and requirement for invasive mechanical ventilation $[4,5]$. There remains a paucity of articles summarizing vascular complications on CT 
angiography (CTA) of the body (especially abdomen-pelvis) and extremities. We aim to describe the spectrum of thromboembolic and hemorrhagic manifestations of COVID-19 infection on CTA body (chest, abdomen-pelvis) and extremity and discuss a review of literature of vascular findings, which, to our knowledge, would be the first of its kind.

\section{Pathophysiology}

SARS-CoV-2 infects host cells by co-opting the angiotensin converting enzyme 2 (ACE2) receptor expressed in the lung, heart, kidney, and bowel; notably, ACE2 is also expressed in endothelial cells. Direct vascular endothelial cell infection by SARS-CoV-2 and subsequent endothelial and inflammatory cell death facilitates the induction of endotheliitis and microvascular dysfunction [6]. This endotheliitis impairs systemic microcirculatory function in vascular beds. In conjunction with subsequent proinflammatory "cytokine storm," with elevated interleukin-2, interleukin-7, granulocyte colony stimulating factor, interferon $\mathrm{Y}$, and tumor necrosis factor $\alpha$, this underlies the development of clinically significant systemic vascular sequelae [6, 7] (Figs. 1 and 2).

Thus, the hypercoagulable state associated with COVID19 infection is putatively due to both direct viral-mediated apoptosis and secondary cytokine storm, microvascular endothelial damage, and hypoxia $[8,9]$. Though the mechanism by which SARS-CoV-2 induces hypercoagulability is incompletely understood, studies suggest that elevated D-dimer in acute COVID-19 infection represents a true

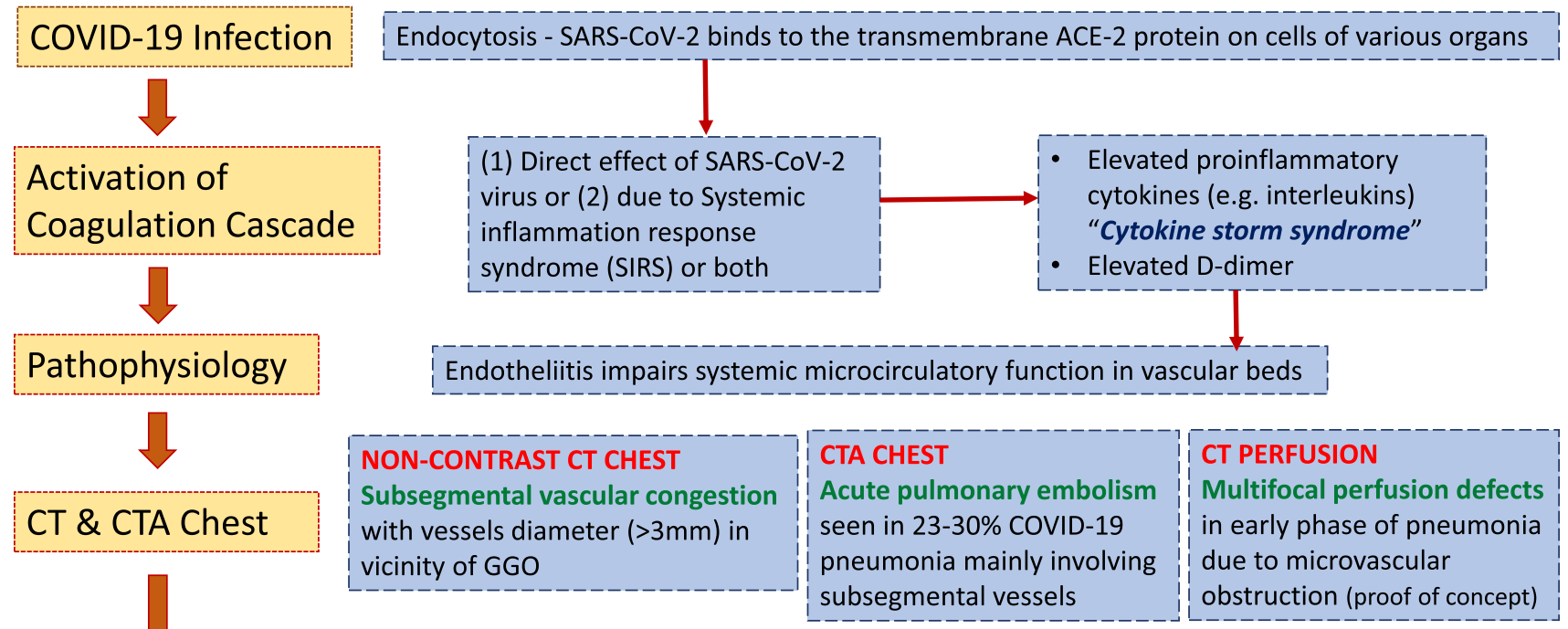

\section{Autopsy Histopathology}

- Diffuse alveolar damage (DAD)

- Pulmonary microvascular thrombosis

Fig. 1: Vascular pathophysiology of COVID-19 and its coagulation cascade consequences

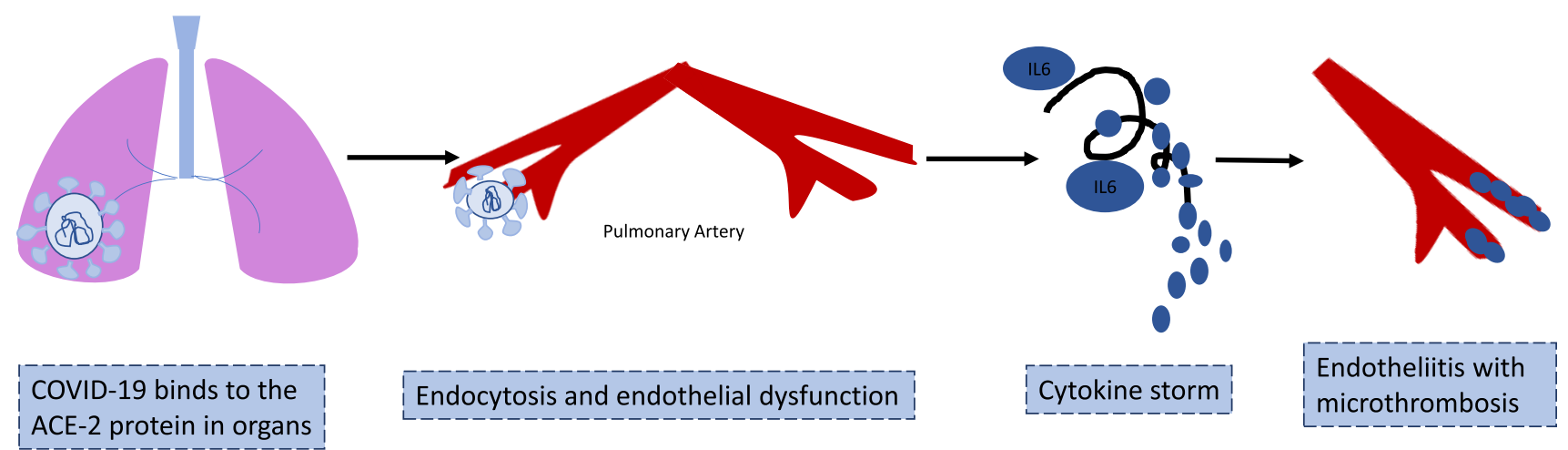

Fig. 2: Cytokine storm-related endothelial sequela of COVID-19 
thrombotic state, i.e., induced by viral-mediated cellular activation and cytokine storm-rather than secondary to systemic inflammation alone. [10].

\section{Review of radiology literature}

A detailed query of PubMed, Google Scholar, and Scopus was performed to identify pertinent publications on CTA findings in the chest, abdomen, pelvis, and extremity of patients hospitalized with COVID-19 infection. The lexicon search terms "coronavirus," "COVID-19," "CTA," and "CT angiogram" were used to identify case reports, case series, and original research publications written in the English language between December 1, 2019, and June 1, 2021, with no restriction on country of study, patient age, race, gender, or number of patients in the study. Initial search identified 323 publications, of which 287 were excluded on inspection of title and abstract for the following criteria: modeling or in vitro studies, studies in non-human subjects, systematic reviews and meta-analyses, articles not written in English, and articles without available full text. An additional 21 publications were removed on detailed review for lack of CT angiogram findings in the areas of interest or lack of vascular findings, leaving 15 publications for inclusion in this review. An additional 8 publications describing vascular findings seen on routine CT scans of abdomen and pelvis were added on secondary review of literature.

We identified a total 23 studies encompassing a total 13,838 patients (Table 1). Of these, 837 patients were critically ill and admitted to the ICU. A total of 513 thromboembolic and 36 hemorrhagic events were documented as detailed in Table 2.

\section{Vascular findings on CT angiography}

\section{CTA chest}

Findings typical of COVID-19 pneumonia have previously been well-described: peripheral-predominant ground-glass opacities (GGO) with and without reticular pattern, consolidation, vacuolar sign, fibrous streaks, vascular dilation, and air bronchogram $[11,12]$. In these patients, acute pulmonary
Table 1 Research publications describing the CTA body and extremity vascular sequelae associated with COVID-19 infections

\begin{tabular}{|c|c|c|c|c|}
\hline No. & Authors & Publication date & Country & $\begin{array}{l}\text { Hospitalized } \\
\text { patients sample size } \\
\text { (ICU) }\end{array}$ \\
\hline \multicolumn{5}{|c|}{ Original Research } \\
\hline 1 & Abdelmohsen et al. & Nov 2021 & Kuwait & 40 (25 ICU) \\
\hline 2 & Vadvala et al. & Jun 2021 & United States & 45 \\
\hline 3 & Cau et al. & May-2021 & Italy & 84 \\
\hline 4 & O'Shea et al. & Apr-2021 & United States & 308 \\
\hline 5 & Valle et al. & Apr-2021 & Italy & 114 \\
\hline 6 & Lee et al. & Jan-2021 & United States & 192 \\
\hline 7 & Horvat et al. & Jan-2021 & Brazil & 81 \\
\hline 8 & Poyiadji et al. & Dec-2020 & United States & 337 \\
\hline 9 & Ogawa et al. & Nov-2020 & United States & 6690 \\
\hline 10 & Baccellieri et al. & Nov-2020 & Italy & 200 (40 ICU) \\
\hline 11 & Ooi et al. & Nov-2020 & United Kingdom & 84 \\
\hline 12 & Moll et al. & Nov-2020 & United States & 210 (102 ICU) \\
\hline 13 & Shah et al. & Sep-2020 & United Kingdom & 187 \\
\hline 14 & Goldberg-Stein et al. & Sep-2020 & United States & 141 \\
\hline 15 & Klok et al. & Jul-2020 & Netherlands & 184 \\
\hline 16 & Lodigiani et al. & Jul-2020 & Italy & 388 (61 ICU) \\
\hline 17 & Al-Samkari et al. & Jul-2020 & United States & $400(144)$ \\
\hline 18 & Grillet et al. & Apr-2020 & France & 100 \\
\hline 19 & Bhayana et al. & Feb-2020 & United States & 134 \\
\hline \multicolumn{5}{|c|}{ Case Series and Case Reports } \\
\hline 20 & Nakamura et al. & Dec-2020 & Japan & 2 \\
\hline 21 & Angileri et al. & Jul-2020 & Italy & 5 \\
\hline 22 & Javid et al. & Jul-2020 & Iran & 1 \\
\hline 23 & Rotzinger et al. & Jun-2020 & Switzerland & 1 \\
\hline
\end{tabular}




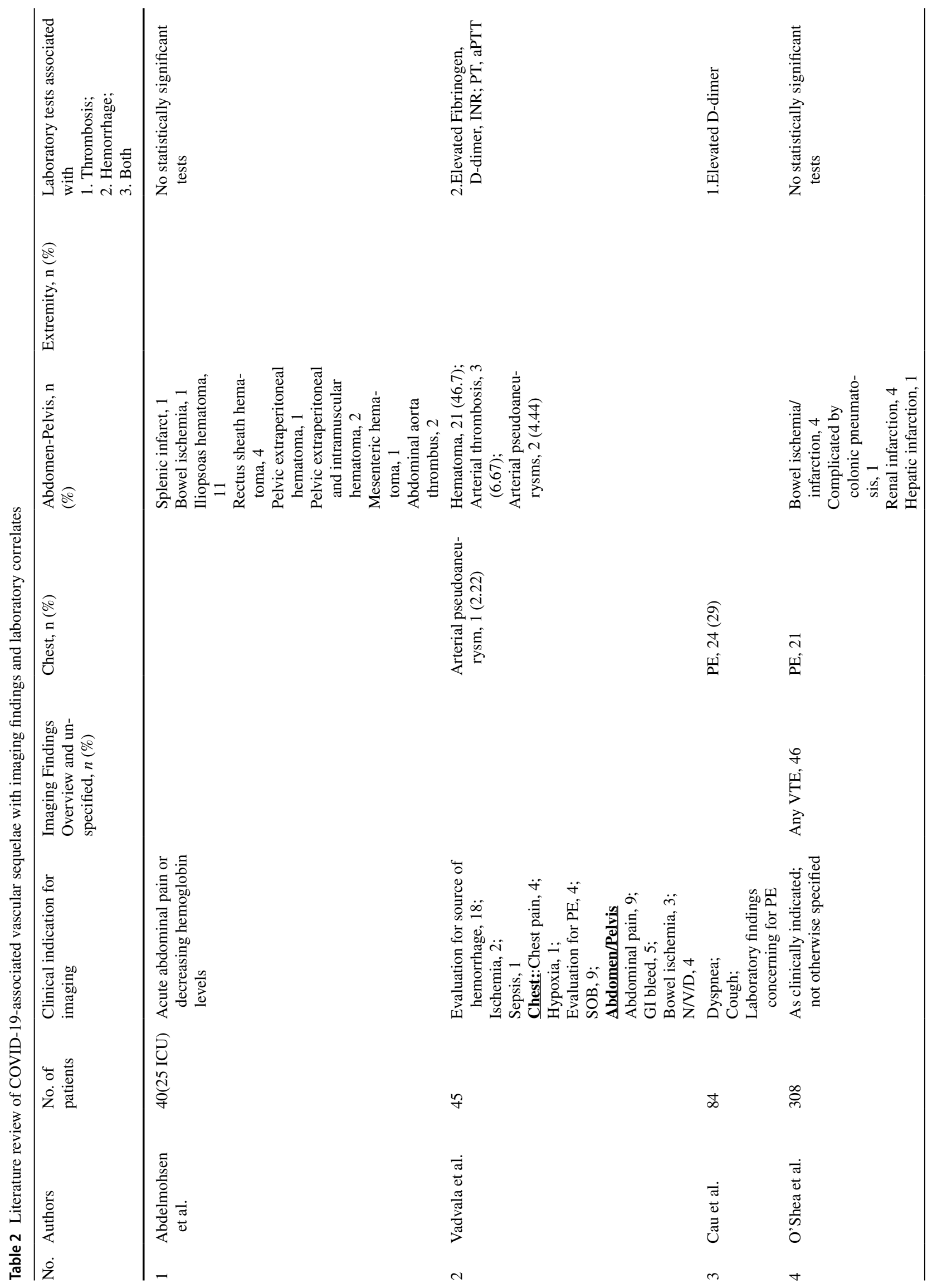




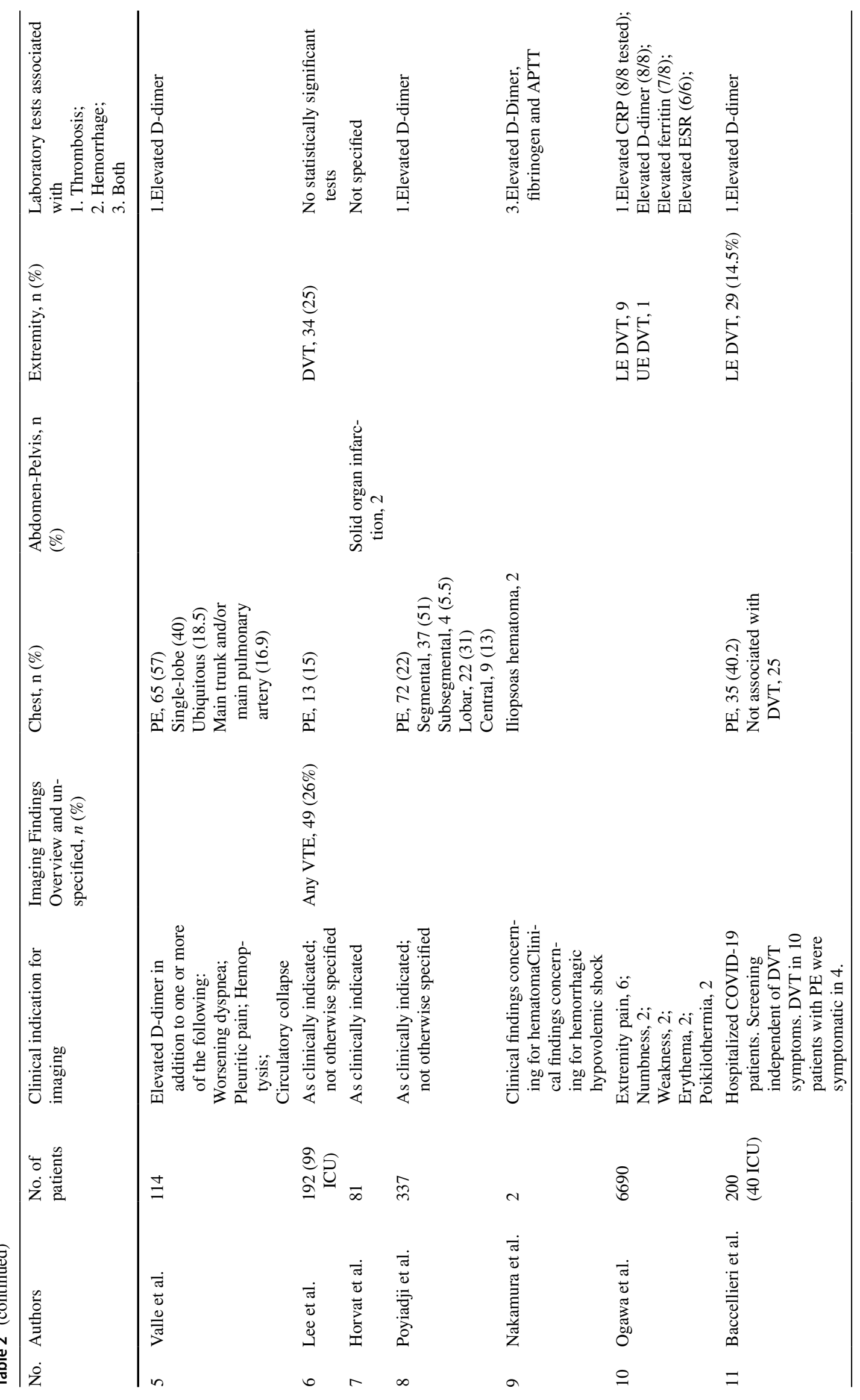




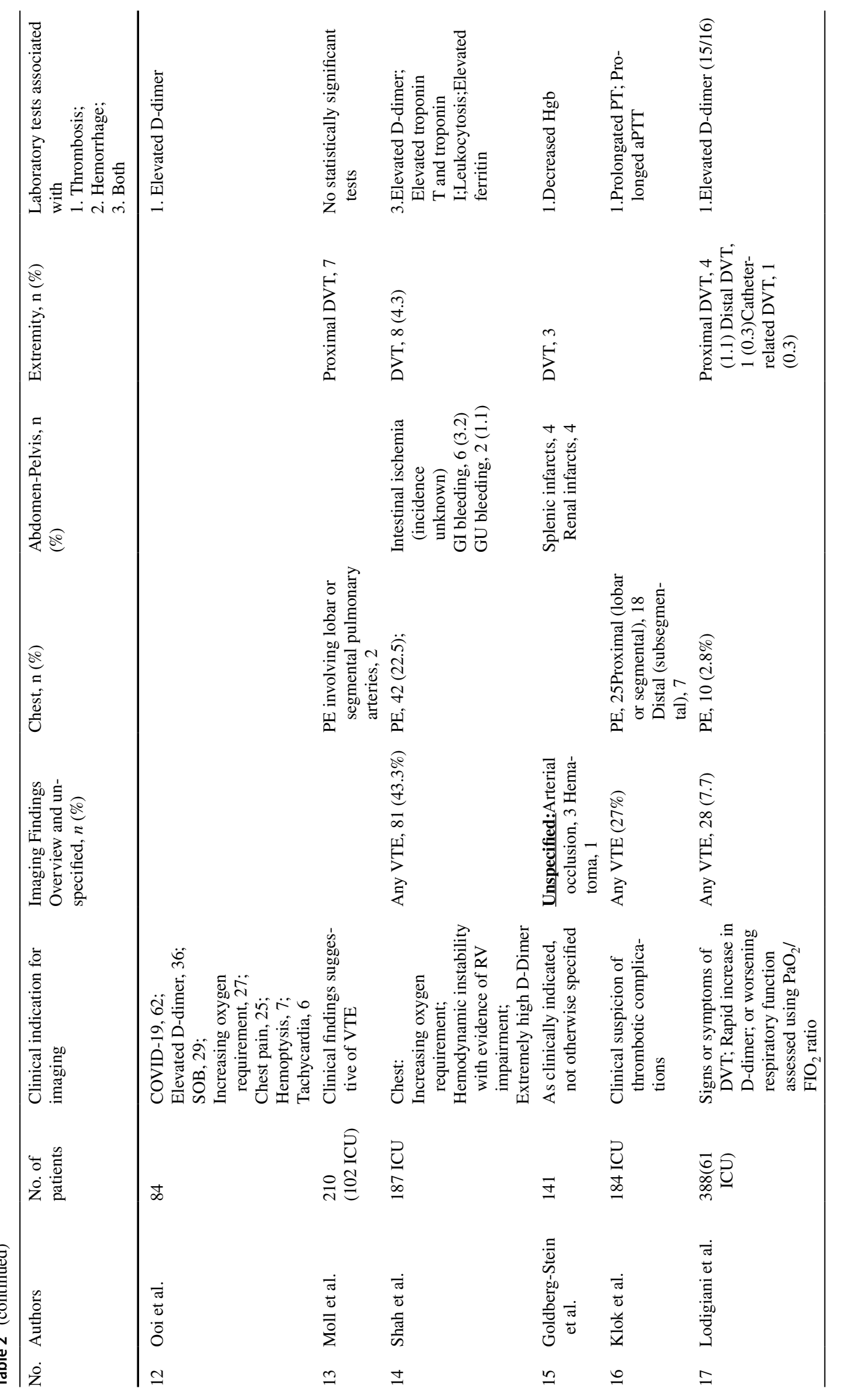




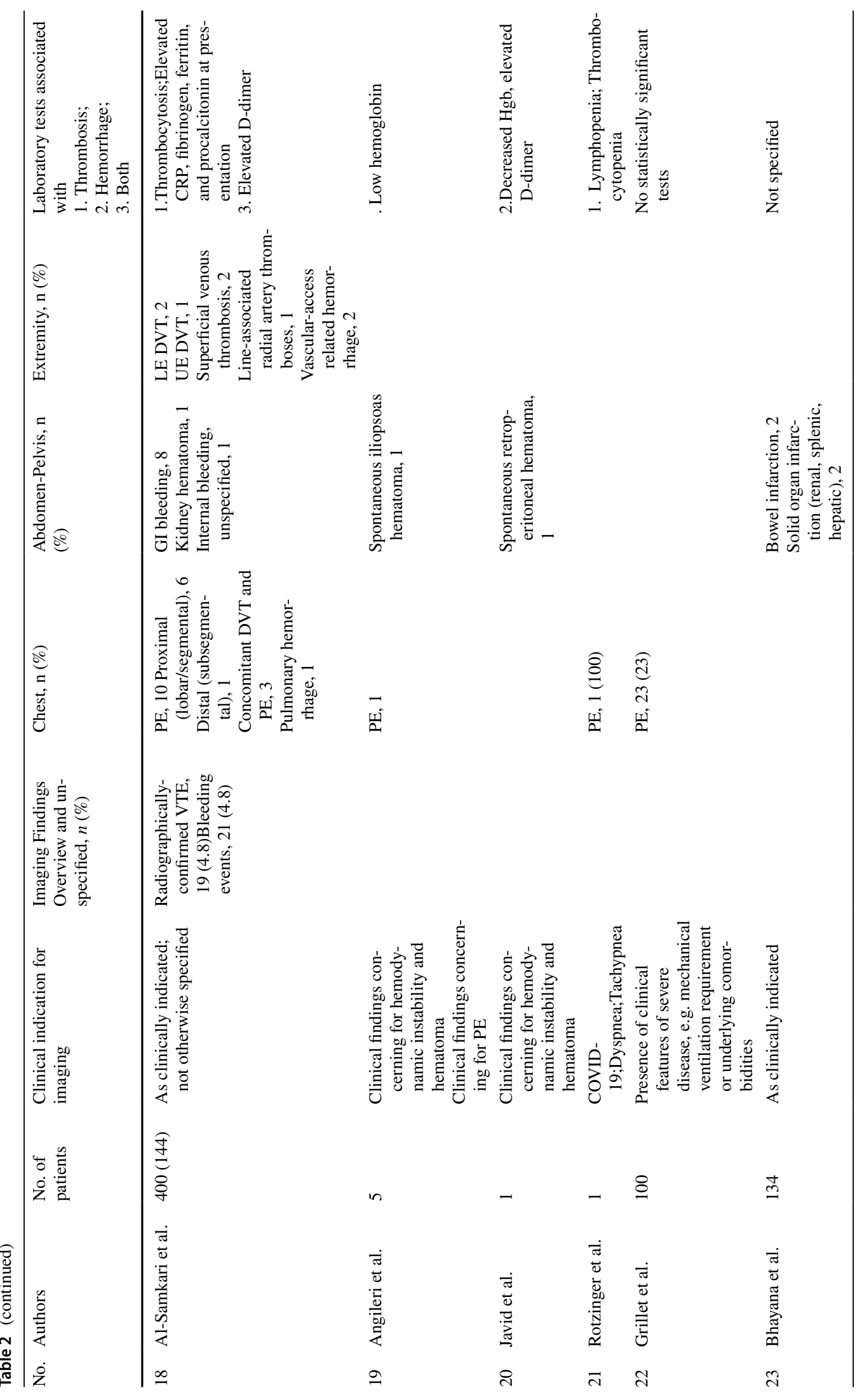


thromboembolism occurs with high incidence, particularly in severely ill, hypoxic patients admitted to the ICU. [13].

\section{Pulmonary embolism}

Subsegmental vascular enlargement (more than $3 \mathrm{~mm}$ diameter) in areas of lung opacity was observed in $89 \%$ of patients with confirmed COVID-19 pneumonia [13]. Pulmonary embolism (PE) is more commonly found in the segmental and lobar branches and less commonly in the central pulmonary arteries. Medium to small vessel dilatation is highly prevalent in COVID-19 pneumonia, is not confined to areas of diseased lung, and often involves subpleural vessels, suggesting a diffuse vascular process. Perfusion abnormalities are common features of COVID-19 pneumonia, including mosaic perfusion, focal hyperemia in a subset of pulmonary opacities, focal oligemia associated with a subset of peripheral opacities, and rim of increased perfusion around an area of low perfusion ("hyperemic halo" sign). [14].

In our review, 12 studies found an incidence of PE ranging from 2.8 to $57 \%$, with patients admitted to the ICU experiencing PE at significantly higher rates [3, 5, 15-24]. Though isolated COVID-19 infection represented the most common indication for CTPA imaging request, clinically apparent dyspnea, hypoxia or increasing oxygen requirement, chest pain, hemoptysis, and tachycardia, as well as elevated D-dimer, were the most common indications for additional imaging [22]. Cau et al. found that COVID-19 patients with radiographically confirmed PE had concomitant increased pulmonary findings of GGO and consolidation, with a worse CT chest severity score and greater extent of lung lobar involvement. Furthermore, most pulmonary emboli (80\%) were found in parenchyma with opacities relating to COVID-19 pneumonia [24]. A majority of PEs occurred in proximal vessels (e.g., lobar or segmental) relative to distal ones (e.g., subsegmental) [17, 21, 25]. Concomitant involvement of other large systemic arteries occurred in a minority of patients [22]. We present several cases of PE associated with aortic thrombus and splenic infarcts (Fig. 3), mycotic pseudoaneurysms of pulmonary artery (Fig. 5), ruptured left renal artery aneurysm (Fig. 6), and central venous line-related DVT of lower extremity veins and psoas hematoma (Fig. 10), as discussed below.

\section{Mycotic pseudoaneurysm}

Mycotic (infected) aneurysms, i.e., aneurysmal degeneration of the arterial wall secondary to bacteremia or septic embolization, are classified as true aneurysms (involving intima, media, and adventitia) or pseudoaneurysms (blind saccular outpouching contiguous with the arterial lumen). Mycotic pseudoaneurysms secondary to COVID-19 infection are exceedingly rare but have been associated with high morbidity and mortality [26, 27]. Imaging findings include multilobulated or saccular aneurysm with or without intraluminal thrombus, intramural air or perivascular presence of fluid,
Fig. 3 A 68-year-old female with recent hospitalization for COVID-19 presented to the emergency department with complaint of abdominal pain. CTA chest abdomen pelvis shows a small, nonocclusive thrombus in aortic arch (red arrow), b small segmental PE in RUL (red arrow), and $\mathbf{c}$ and $\mathbf{d}$ multiple wedge-shaped splenic infarcts in axial and coronal images (red arrow)
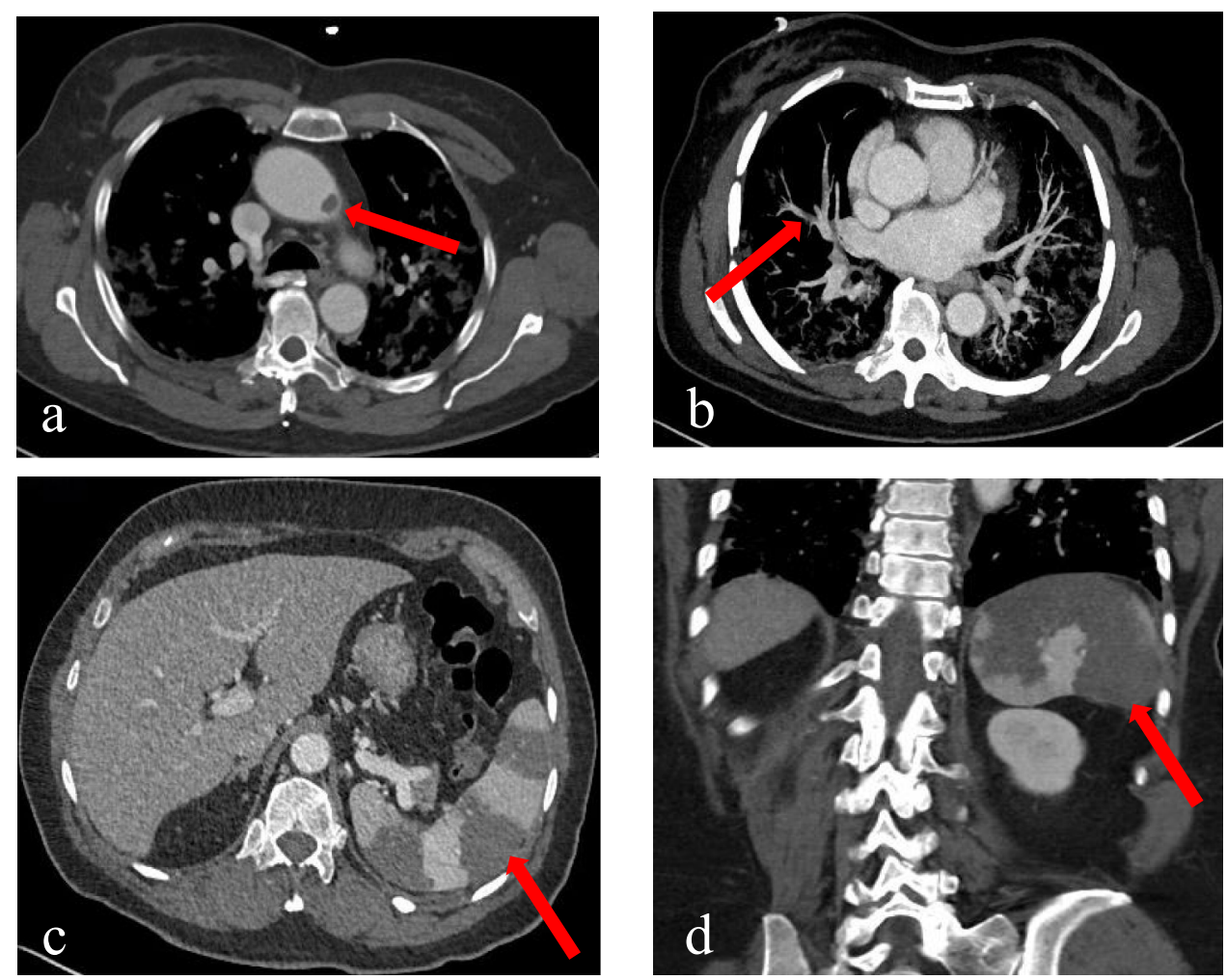
Fig. 4 A 76-year-old male was hospitalized due to COVID19 pneumonia. a CT abdomen pelvis 1 year ago showed multifocal calcified and small noncalcified atherosclerotic plaques (red arrow). b CTA 1 year later, during admission for COVID-19, demonstrated significant interval increased soft atherosclerotic plaque/ intramural thrombus of the abdominal aorta causing mild stenosis (red arrow)
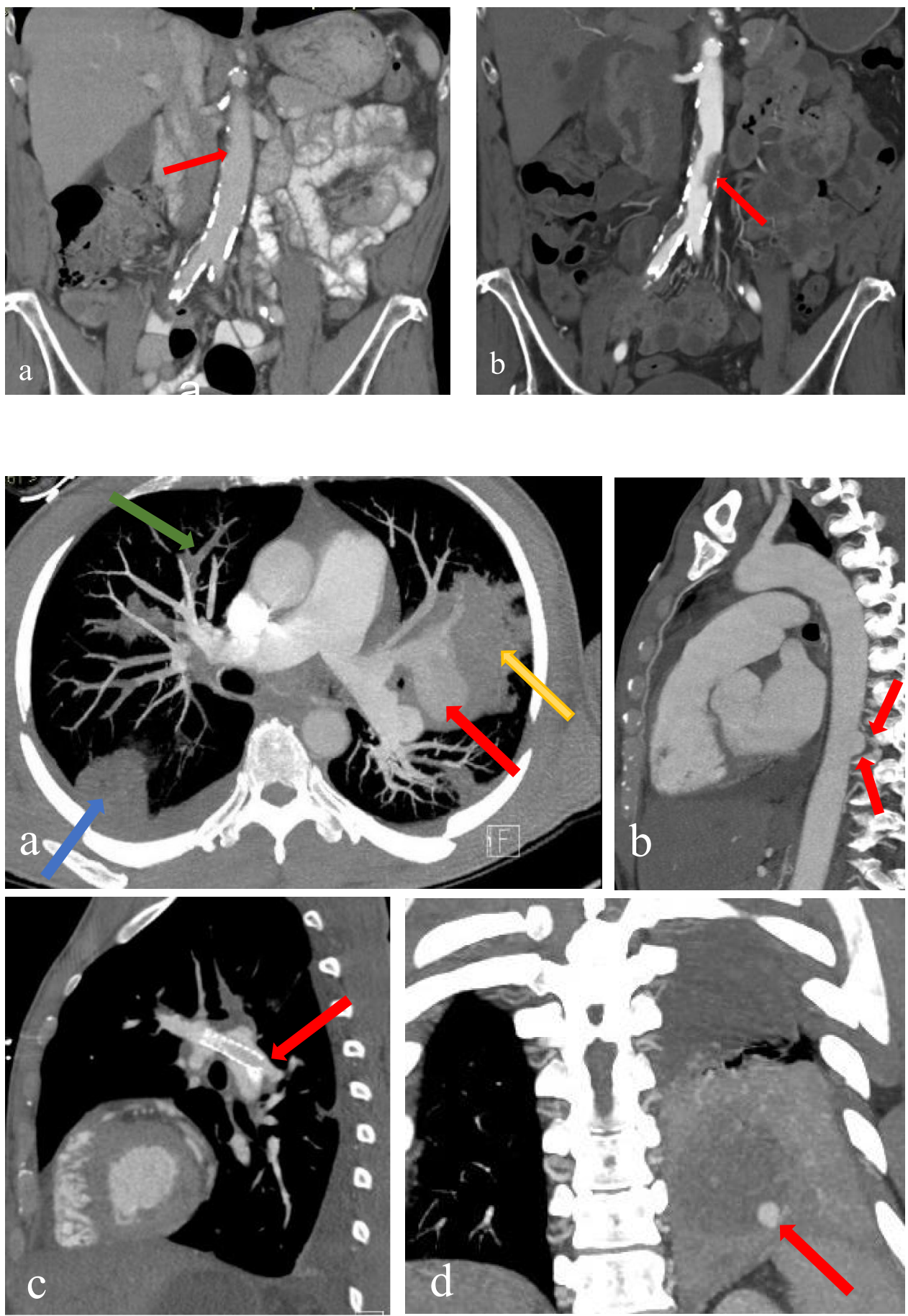

Fig. 5 A 52-year-old male was hospitalized with COVID19. CTA chest shows a large multilobulated pseudoaneurysm of left lobar pulmonary artery in the upper lobe (red arrow) with associated hemorrhage suggestive of rupture (yellow arrow). Segmental PE in the right upper lobe pulmonary artery (green arrow) and wedgeshaped pulmonary infarct in right lower lobe (blue arrow). b Small saccular descending acic aorta pseudoaneurysm and Amplatzer plug placement in pseudoaneurysm of left lobar pulmonary artery. $\mathbf{d}$ A separate patient, 35-year-old male, was admitted with COVID-19 pneumonia that got complicated by necrotizing pneumonia in left lower lobe, with associated small pseudoaneurysm arising from segmental left lower lobe pulmonary artery soft tissue stranding, and/or air (Fig. 5). These pseudoaneurysms may be complicated by rupture and hemorrhage if not diagnosed and treated promptly. We present a case of bilateral central PE complicated by pseudoaneurysm formation and hemorrhage in the left lobar pulmonary artery. This patient was managed with pulmonary artery stents and Amplatzer plug placement which ultimately achieved stasis and subsequent thrombosis of pseudoaneurysm. In another case (Fig. 5d), a young male who was admitted with COVD19 pneumonia complicated by necrotizing pneumonia with associated small lung parenchymal pseudoaneurysm likely arising from the subsegmental branch of left lower lobe pulmonary artery. This patient was managed with left lower lobectomy. 
Fig. 6 A 59-year-old female with COVID-19 pneumonia and numerous complications presented with a drop of hemoglobin from 14 to $7 \mathrm{~g} / \mathrm{dl}$. CTA scan shows a large left perinephric hematoma due to ruptured $9 \mathrm{~mm}$ aneurysm of left renal arcuate artery with active extravasation in the upper pole of the left kidney (red arrow), b large left perinephric retroperitoneal hematoma (3D VRT) (red arrows), c active extravasation confirmed on selective left renal angiogram and managed with coil placement (red arrow), and d non-occlusive pulmonary embolism in left lower lobar pulmonary artery (yellow arrow) and ruptured aneurysm of left renal arcuate artery (red arrow)
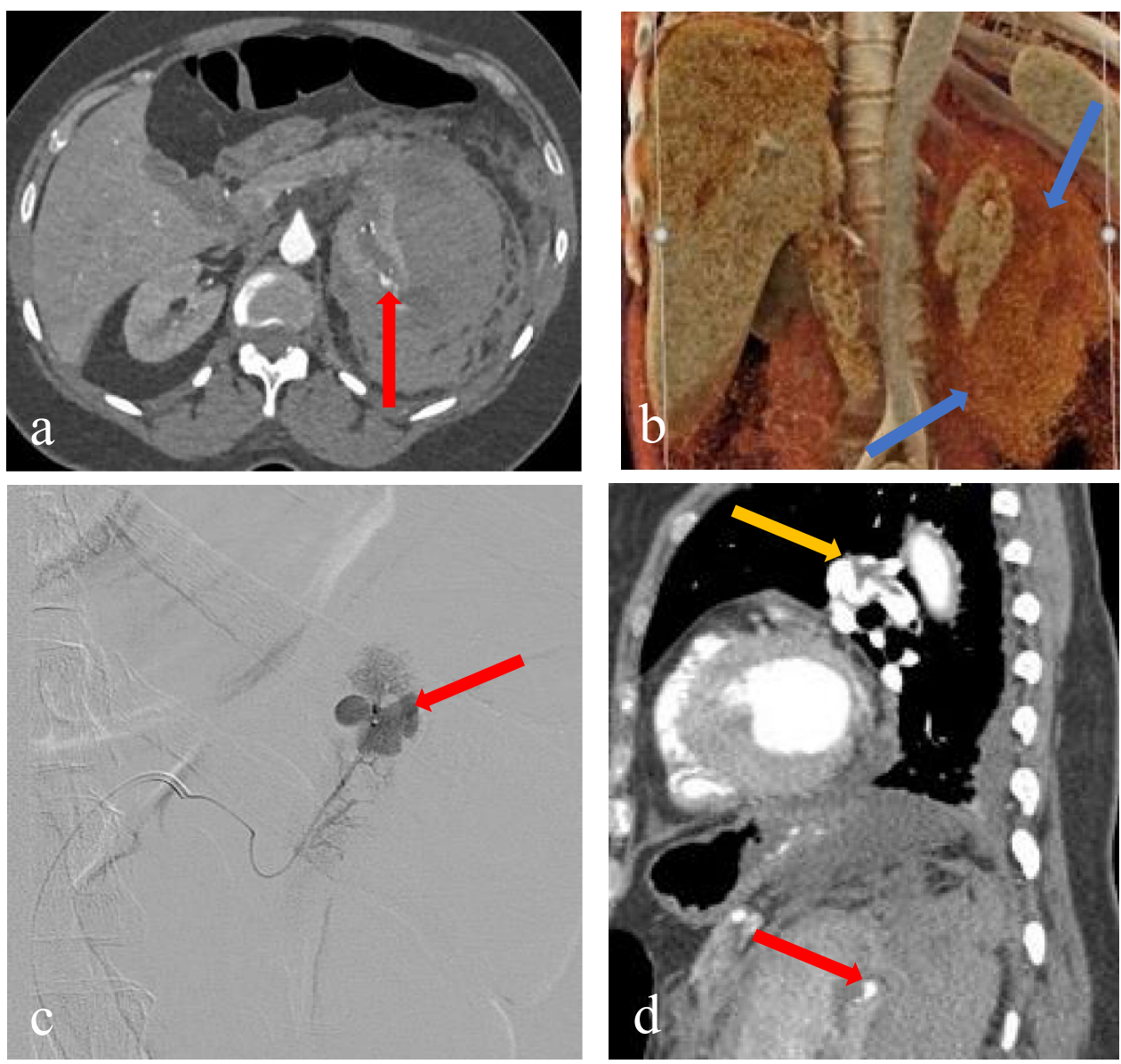

\section{Aortic thrombosis}

Aortic thrombosis is a rare complication of COVID-19 infection and can present asymptomatically or with a consequence of ischemic events. A number of case reports and series have discussed free-floating aortic thrombi in the setting of COVID-19 infection without associated atherosclerosis. In one case, CTA demonstrated an aortic thrombus at the aortic arch without definitive aortic atherosclerosis and associated with splenic infarcts and pulmonary embolism (Fig. 3). In another case, a COVID-19 patient presented with significantly enlarged size of known atherosclerotic plaques in the infrarenal abdominal aorta as compared to prior CT scan 1 year ago suggestive of accelerated process of intimal injury and atherosclerotic soft plaque deposition causing mild aortic stenosis (Fig. 4). These patients are usually managed with anticoagulation therapy as a standard of care unless vascular intervention such as thromboembolectomy is required for larger occlusive thrombus in order to prevent emboli shower [1, 28, 29]. Although rare, these aortic thromboses have also been associated with occlusion of distal end arteries affecting organs such as the kidney and spleen [29-31] (Fig. 3).

\section{CTA abdomen and pelvis}

The most common indication for acquisition of CTA was abdominal pain, followed distantly by complaint of vomiting and fever. Over half of patients who underwent CT have positive findings, most commonly nonspecific intestinal distension, colorectal and small bowel mural thickening, gallbladder wall thickening, and ascites [32, 33]. By comparison, vascular findings are relatively uncommon as compared to non-vascular findings. COVID-19 patients present with both thrombotic and hemorrhagic adverse events with hemorrhagic events being more common [34]. Hematomas involving the retroperitoneum, abdominal wall, gluteal regions, or upper thigh are the most commonly reported hemorrhagic complication [34-36]. Thrombotic events demonstrated on CTA include both occlusion of major arteries and solid organ infarct including, but not limited to, the intestinal tract, kidneys, pancreas, and liver. [32, 34, 37].

\section{Mesenteric ischemia/infarction}

Bowel ischemia/infarcts have been described as a rare complication overall in COVID-19 patients but is a more 
common vascular consequence in the abdomen and pelvis [20]. CTA may demonstrate pneumatosis intestinalis, pneumobilia, pneumatosis portalis, pneumoperitoneum, and variable reactive peritoneal free fluid. Very rarely, vascular occlusion or vasculitis of mesenteric arteries such as branches of celiac axis or superior mesenteric artery (SMA) may be detected. An abdominal-pelvic findings study by Goldberg-Stein demonstrated 15\% bowel wall thickening, out of which $6.2 \%$ were found in the colon; although mural wall thickening is a non-specific finding, it is suggestive of enteritis or colitis inclusive of microvascular ischemic changes. [32].

\section{Solid organ infarction and bleeding}

Hepatic, renal, and splenic ischemia have been described in association with patients critically ill with COVID-19 [20]. A CT abdomen-pelvis study conducted by Goldberg-Stein found 5\% incidence each of splenic and renal infracts out of 141 patients [32]. CTA demonstrates hypoenhancement and wedge-shaped cortical hypodensity of variable sizes (Fig. 3). Infarcts may be large and diffuse, involving the entire organ, or multifocal within a single organ. These are typically acute infarcts and hence might present with organ swelling with hypoenhancement in the early phase of microinfarction and then later might progress to loss of organ volume in the subacute to chronic infarction phase.

Intrapenchymal bleeding within solid organs is less common than infarct and is a rare presentation. We present a case of active bleeding from a ruptured aneurysm of arcuate branch of left renal artery causing large hematoma at upper pole of left kidney extending into retroperitoneum (Fig. 6).
There was a concurrent non-occlusive pulmonary embolism in left lower lobar pulmonary artery. Active bleeding was managed with coil placement during superselective left renal artery angiogram.

\section{Gastrointestinal bleeding}

Gastrointestinal hemorrhage has commonly been described in association with acute COVID-19 infection. We present one such case of active arterial bleeding involving rectum and associated large sigmoid colonic hematoma; the arterial bleeder was arising from inferior rectal branch of internal iliac artery (Fig. 7). While data is limited on which risk factors, patient characteristics, and clinical courses are associated with increased risk of gastrointestinal hemorrhage in COVID-19 patients, some information has been reported. In a meta-analysis of 663 COVID-19 patients, of which $34 \%$ presented with gastrointestinal bleeding, Chen et al. found that history of gastrointestinal bleeding was moderately to highly correlated with history of oral anticoagulation [38]. COVID-19 patients who presented with gastrointestinal bleeding had higher mortality rates and had higher risk of gastrointestinal ulcers versus esophagitis on upper GI endoscopy [38]. Goyal et al. further demonstrated that most COVID-19 patients with gastrointestinal bleeding were conservatively managed with therapeutic endoscopy required in only $12.2 \%$ of cases and blood transfusion required in approximately $50 \%$ of patients [39]. Notwithstanding, some acute gastrointestinal bleeding in unstable patients do require vascular intervention.
Fig. 7 A 57-year-old male with no past medical history is hospitalized with COVID-19 pneumonia and ARDS. CTA scan shows active arterial bleeding along the anterior and right walls of the rectum from inferior rectal artery (red arrows in $\mathbf{a}, \mathbf{b}$, and $\mathbf{c}$ ), intraluminal sigmoid hematoma (yellow arrows in $\mathbf{b}$ and $\mathbf{c})$, and small intramuscular hematoma in right gluteus maximus (blue arrows in $\mathbf{b}$ )
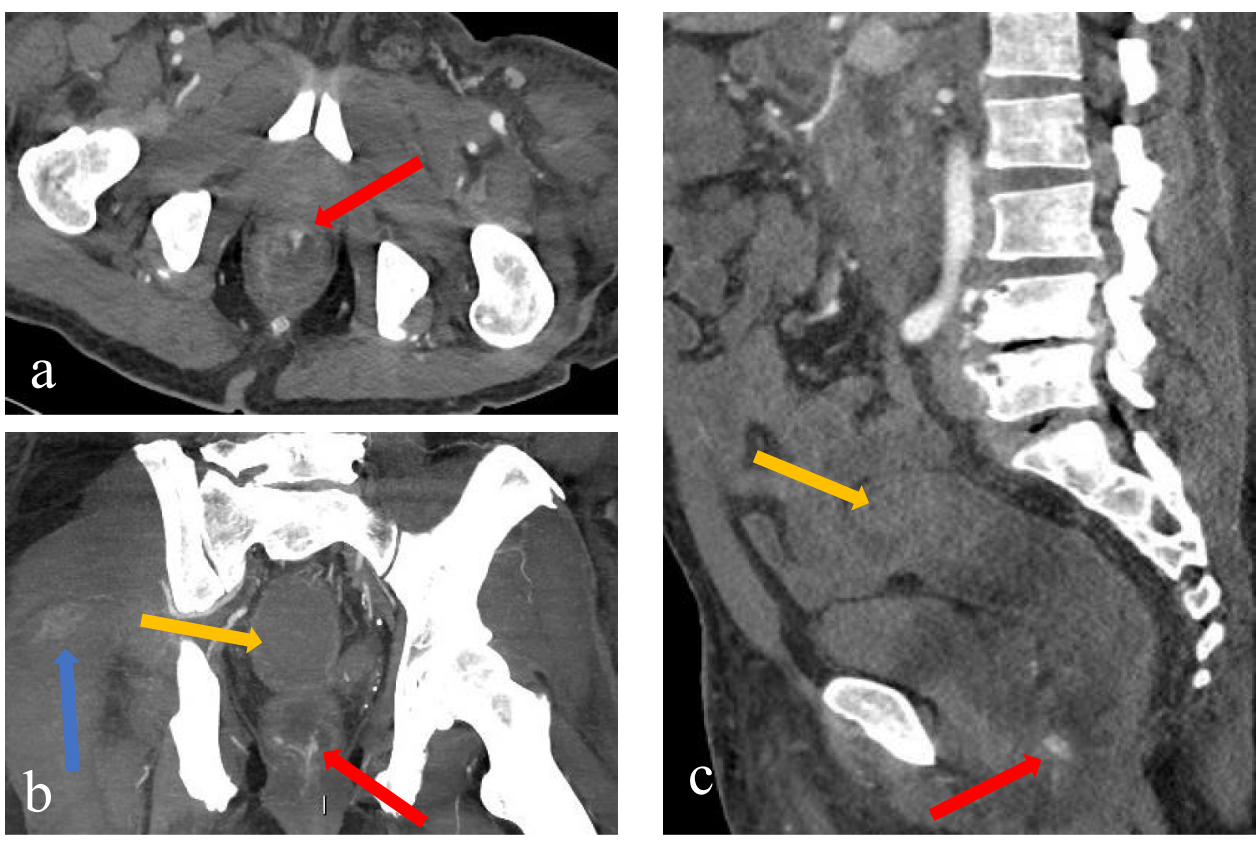


\section{Intramuscular and retroperitoneal hematoma}

COVID-19-associated small-vessel hemorrhage resulting in musculoskeletal system involvement, notably intra- and peri-muscular hematoma, has been well-described. The most common hematoma site is the iliopsoas involving the retroperitoneum, followed by rectus sheath involving the anterior abdominal wall, pelvic hematoma, hemoperitoneum, and subcapsular organ hematomas $[32,34-36$, 40-42] (Figs. 7-10). Imaging findings include variable size hyperdense mass like hematoma often with blood-fluid hematocrit level depending on the timing of bleed, causing displacement and compression of surrounding structures. If the hematoma is huge, there is also risk of compartment syndrome, more commonly in tighter spaces like extremities and less likely within the abdominal cavity. A subacute hematoma might appear hypodense due to evolution of blood products accumulated over a period of time.

\section{CTA extremity}

\section{Deep venous thrombosis}

Deep vein thrombosis is a prevalent finding in patients with severe COVID-19, with ultrasonography as a primary method of diagnosis. Though true incidence is unknown, Pieralli et al. detected DVT in 13.7\% of COVID-19 pneumonia patients screened on ultrasound surveillance protocol; most of these patients were asymptomatic at time of screening. An ultrasound study conducted by Gawande et al. found that out of a total 196 US studies, extremity venous doppler US was the most commonly requested study (51\%), and the most common finding was deep vein thrombosis in $18.1 \%$ of studies. [43].

Though bedside ultrasonography (or Doppler) remains a mainstay of initial diagnosis of DVT in the extremities, and has been shown to be diagnostically equivalent to CT venography (CTV), CTV can be performed in patients for whom there is a high clinical suspicion for DVT with simultaneous PE in concert with CTPA without requiring additional contrast material [44]. This provides both direct visualization of thrombi and increased sensitivity in detecting occlusion of thoracic and proximal abdominopelvic veins typically undetectable by US. Chen et al. found significantly lower incidence of DVT on US (4.3\%) compared to CTV (82.6\%) in patients with mild or moderate COVID-19 pneumonia [45]. The increased sensitivity of CTV compared to doppler US provides validation for the implementation of CTV as an effective and sensible diagnostic modality.

On CTV, typical features of DVT include contrast filling defect with hypodense thrombus, venous distension, rim enhancement of the vein, and perivenous edema or fat stranding (Figs. 8 and 10). Often times DVT in extremities is accompanied by intramuscular hematomas. This combination of thrombosis and hemorrhage is challenging to manage and hematology experts are consulted for titration of anticoagulants and blood transfusion as indicated.

\section{Peripheral arterial thrombosis}

Acute thrombosis of peripheral arteries is a rare but critical finding in hospitalized COVID-19 patients. The odds are higher due to hypercoagulable state, relative immobility, and increased vascular access requirement in hospitalized COVID-19 patients. Ogawa et al. first illustrated large vessel peripheral extremity occlusion in association with findings of COVID-19 pneumonia in a case series of 9 patients, even in absence of preexisting hypercoagulable state or calcific atherosclerotic disease [46]. The authors found that there was no correlation between the length of arterial occlusion and the severity of COVID-19 pneumonia. A longer length of about $10 \mathrm{~cm}$ arterial occlusion was seen in patients with lower extremity involvement. They also found that a pre-existing calcified atherosclerotic plaque within the vessel wall was not necessarily contributing to increased risk of thrombotic arterial occlusion. Both Ogawa et al. and Goldman et al. showed that due to great thrombus burden in extremity arteries there is increased likelihood of open thrombectomy, amputation, or death in patients with COVID-19. [47] We present a case of acute non-occlusive arterial thrombus in the proximal right common femoral artery with associated similar characteristic thrombus in the abdominal aorta. This patient underwent right iliofemoral thromboendarterectomy with bovine pericardial patch angioplasty during initial admission which was complicated by a large postoperative pseudoaneurysm (Fig. 9). This patient also had an acute thrombus formed in the abdominal aorta, which might have been the source of embolism to the right lower extremity.

\section{Vascular access-related complications}

Due to immediate viral- and cytokine-mediated vascular changes, COVID-19 patients are at particularly high risk of complications from otherwise low-risk interventions. Though uncommon, vascular access-related complications represent an underrecognized source of morbidity in these vulnerable patients.

\section{Catheter-related thrombosis}

In a large-scale retrospective study of vascular access in COVID-19 patients, Gidaro et al. found catheter-related thrombosis (CRT) occurred in $42(1.9 \%)$ of cases [48]. In other studies, most identified cases occurred in peripheral veins and in the context of hemodialysis [49-54]; rare cases 
Fig. 8 A 49-year-old male was admitted for COVID-19 pneumonia developed right lower extremity swelling. CTA bilateral lower extremity showed a nearly occlusive DVT involving the left common femoral vein (blue arrow), b right thigh hematoma involving quadriceps femoris with active extravasation (red arrow), c right thigh hematoma with hematocrit level (red arrow) and bilateral femoral vein DVT (blue arrows), and d large right thigh hematoma (red arrow) and right external iliac vein DVT (blue arrow)
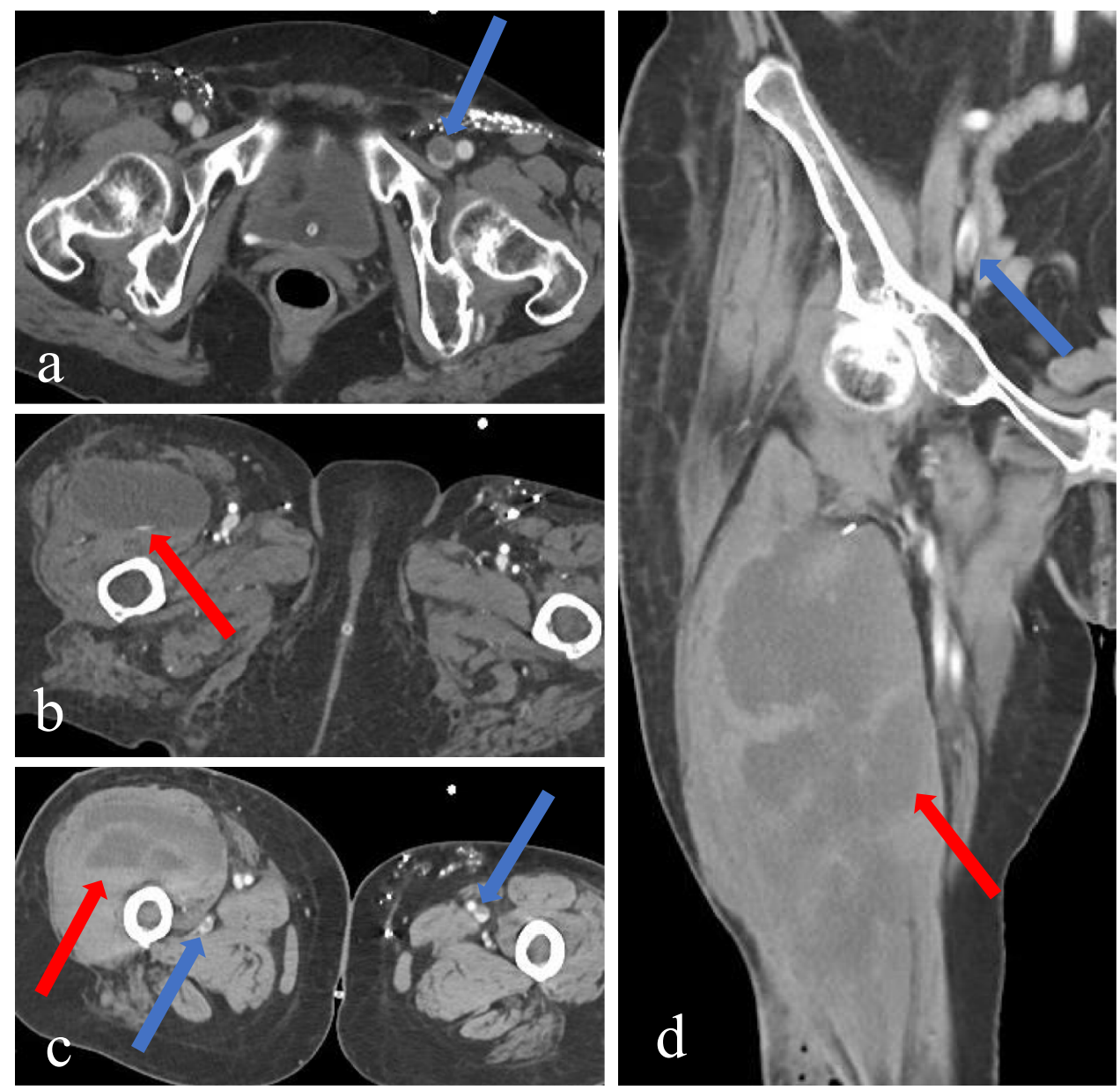

of CRT secondary to central venous catheter thrombosis have also been described $[55,56]$. Though vascular access devices are especially prone to thrombosis, patients with a requirement for long-term vascular access may benefit from a low-dose heparinized saline protocol. [57].

We illustrate a case of venous line-related thrombus in the right common femoral vein and infrarenal IVC; note inhomogeneous thrombus adhering to the central venous line partially occluding the veins involved (Fig. 10). This same patient had concurrent left psoas hematoma and segmental PE. The patient was initiated on anticoagulation but unfortunately passed away a week after the scan.

\section{Pseudoaneurysm related to intervention}

Iatrogenic pseudoaneurysm is an exceedingly rare complication; Al-Thani et al. presented one case of subclavian artery pseudoaneurysm induced by central venous catheter insertion [58]. The vessel wall weakness due to underlying endotheliitis secondary to both the COVID-19 virus itself in addition to vascular intervention/procedures might be the causative factor. We present a rare case of a large pseudoaneurysm of the right common femoral artery secondary to thrombectomy (Fig. 9). A careful scrutiny of vasculature should be performed on CTA to look for vessel wall irregularities and small outpouchings as these can lead to catastrophic bleeding events.

\section{Imaging summary}

\section{Thromboembolism}

In most studies, venous and arterial thromboembolic events were more frequently described than hemorrhagic events. Furthermore, thromboembolic events are described to occur with greater frequency in ICU patients. Pulmonary embolism and lower extremity deep vein thrombosis were the most frequently described thrombotic events, followed distantly by consequence of bowel ischemia and solid organ infarcts [3, 5, 15-24, 32, 33, 37, 41, 59]. Since thrombotic events are more common, they frequently co-exist with hemorrhage, as seen in several of the examples in this article. It is important to recognize deep vein venous thrombosis, especially in the periphery, in a timely manner in order to avoid a dreadful complication of a large central PE. 

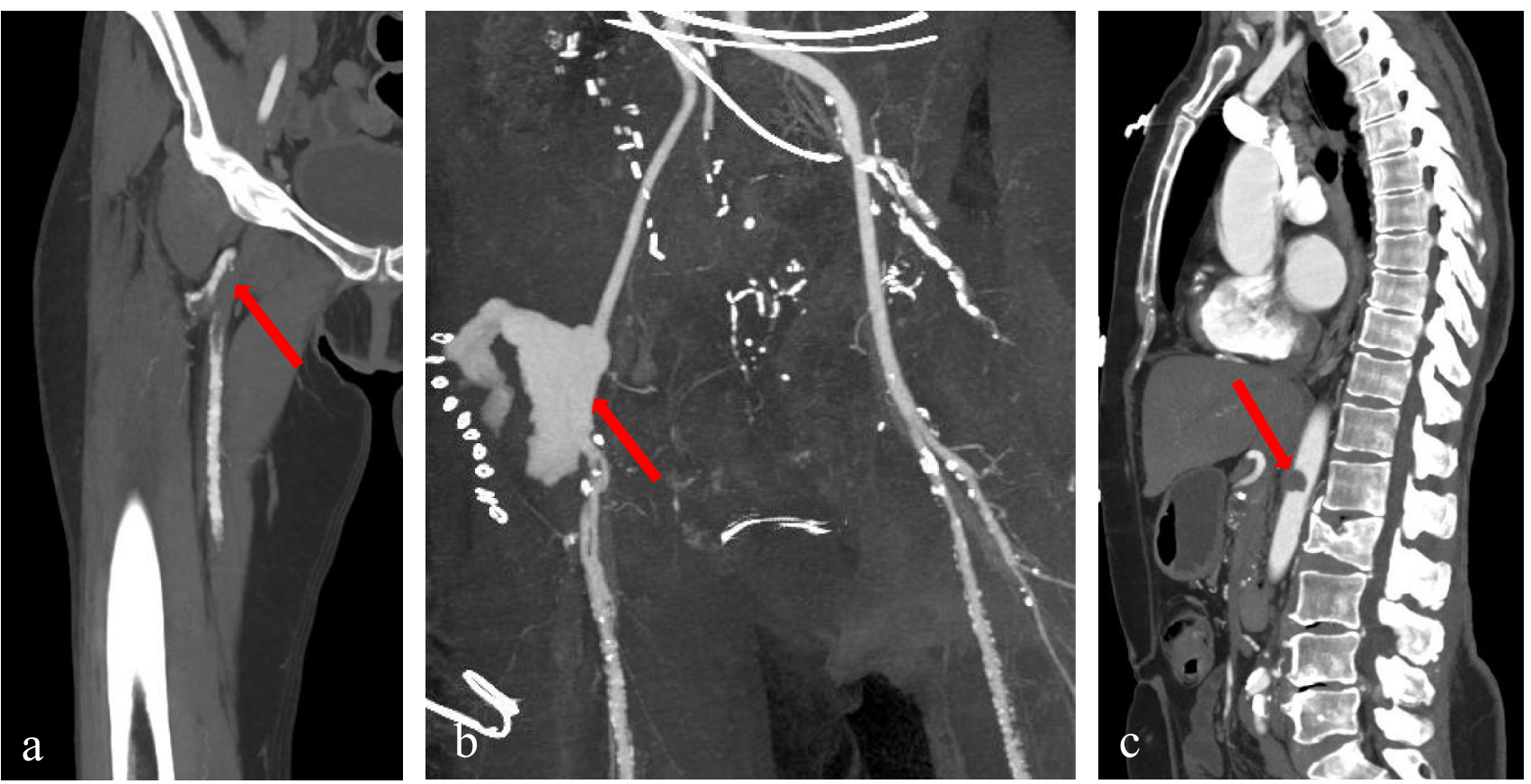

Fig. 9 A 66-year-old female initially presented to the emergency department with complaints of new onset right foot pain. CTA scan shows a CTA right lower extremity demonstrated acute, nearly occlusive thrombus in the proximal right common femoral artery and superficial femoral artery (red arrow). Patient underwent right ili- ofemoral thromboendarterectomy with bovine pericardial patch angioplasty which got complicated by $\mathbf{b}$ dehiscence of common femoral artery patch and large pseudoaneurysm (red arrow) and c concurrent non-occlusive acute thrombus in abdominal aorta (red arrow)
Fig. 10 A 67-year-old male COVID-19-positive patient presented with abdominal pain and was found to have pancreatitis (not shown here). CT abdomen pelvis shows a central vein line-associated DVT of right common femoral vein (red arrow), b large left psoas hematoma (red arrow), c central vein line-associated small thrombus in distal IVC (red arrow), and $\mathbf{d}$ small segmental PE in left lower lobe pulmonary artery branch (red arrow)
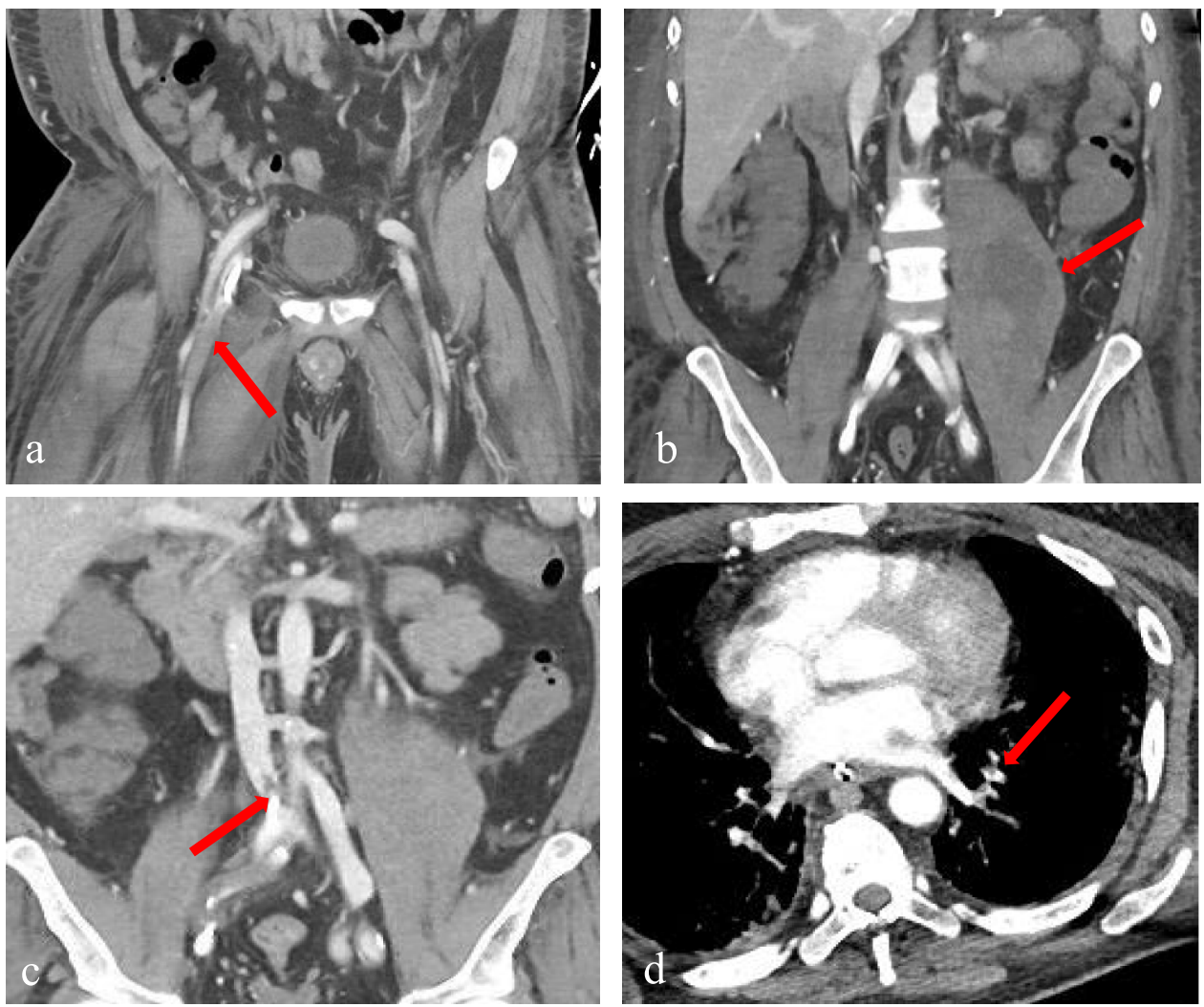


\section{Hemorrhage}

Clinically significant bleeding events occurred primarily in ICU patients, with incidence between 7.6 and 8\%, compared to $1.9 \%$ in patients on floor units $[3,4,25]$. Of these events, $4.8 \%$ were classified as major bleeding (World Health Organization grades 3 and 4), with at minimum gross blood loss requiring transfusion [3]. In patients receiving prophylactic or therapeutic anticoagulation, hemorrhage commonly occurs and may affect multiple organ systems in the body, with gastrointestinal bleeding being the leading cause of hemorrhagic events, followed by spontaneous intramuscular hematomas. Other hemorrhagic events that have been infrequently described in association with COVID-19 infection include epistaxis, oral/oropharyngeal bleeds, hemoptysis, pulmonary hemorrhage, cerebral hemorrhage, and kidney hematoma $[2,25]$. Though an uncommon finding, active arterial bleeding may indicate a life-threatening hemorrhage and thus requires rapid identification on contrast-enhanced CT.

\section{Conclusion and recommendation}

Vascular complications including both thromboembolism in the setting of hypercoagulability and bleeding in the setting of prophylactic anticoagulation are clinically significant concerns for critically ill COVID-19 patients. Commonly affected regions are the pulmonary artery, GI tract, kidney, hepatobiliary organs, extremity vessels, and aorta. Thus, the decision to initiate anticoagulation must be weighed against concern for bleeding risk, and patients should be carefully monitored for possible hemorrhage. It is important that all radiologists and clinicians are aware of the vascular (thromboembolic and hemorrhagic) complications on CTA body and extremity of COVID-19 patients in order to provide timely diagnosis and guide appropriate management.

\section{Image acquisition technique}

Select CTA chest and CTA abdomen pelvis scans that we present were acquired via SOMATOM Sensation 64-slice helical CT scanner or SOMATOM Definition Flash 128slice helical CT scanner, Siemens Healthineers, Forchheim, Germany. Identical CTA protocols were utilized for both scanners.

A dual phase scan following intravenous power injection of 100-120 mL Omnipaque 350 or Visipaque 320 IV nonionic contrast dye (based on patient weight) at the rate of 4-5 mL/s was performed for all CTA scans, acquiring arterial phase between $25-30 \mathrm{~s}$ and portal venous phase between 50-70 s. Whenever possible, water (as a negative contrast) was given orally to obtain luminal distention of the stomach and small bowel. Reconstruction of $3 \mathrm{~mm}$ thick axial slices and $0.75 \mathrm{~mm}$ thin axial slices was performed for both arterial and venous phases, with B20f and B80f reconstruction algorithms used for soft tissue and lungs, respectively. Postprocessing was performed to obtain coronal and sagittal reformats as well as vascular mapping using 3D MIP and volume rendering.

Lower-extremity images, where indicated, were acquired with extremity runoff, wherein a bolus-triggered arterial phase was acquired as per above protocol, with bolus tracking performed off the abdominal aorta using a $230 \mathrm{HU}$ trigger setting. 3D postprocessing reconstruction B30f was performed for soft tissue reformats.

Abbreviations ACE2: Angiotensin converting enzyme 2; COVID19: Coronavirus disease 2019; CRT: Catheter-related thrombosis; CTA: Computed tomography angiography; CTPA: CT pulmonary angiogram; CTV: CT venography; DIC: Disseminated intravascular coagulation; DVT: Deep vein thrombosis; GGO: Ground-glass opacities; ICU: Intensive care unit; IVC: Inferior vena cava; PE: Pulmonary embolism; SARS-CoV-2: Severe acute respiratory syndrome coronavirus-2; US: Ultrasound

Acknowledgements The data utilized for this publication were part of the JH-CROWN: The COVID PMAP Registry which is based on the contribution of many patients and clinicians. We are thankful to the JH-CROWN registry.

Availability of data and material The JH-CROWN registry.

Code availability Not applicable.

\section{Declarations}

Ethics approval Yes. IRB approved.

Consent to participate An informed consent was waived by the IRB for this retrospective study.

Conflict of interest The authors declare that they have no conflict of interest.

\section{References}

1. N T, H B, X C, J G, D L, Z S. Anticoagulant treatment is associated with decreased mortality in severe coronavirus disease 2019 patients with coagulopathy. J Thromb Haemost. 2020;18(5):1094 1099. https://doi.org/10.1111/JTH.14817

2. Musoke N, Lo KB, Albano J et al (2020) Anticoagulation and bleeding risk in patients with COVID-19. Thromb Res 196:227. https://doi.org/10.1016/J.THROMRES.2020.08.035

3. Shah A, Donovan K, McHugh A, et al. Thrombotic and haemorrhagic complications in critically ill patients with COVID19: a multicentre observational study. Crit Care 2020241. 2020;24(1):1-10. https://doi.org/10.1186/S13054-020-03260-3 
4. I P, V F, A L, et al. Association of treatment dose anticoagulation with in-hospital survival among hospitalized patients with COVID-19. J Am Coll Cardiol. 2020;76(1):122-124. https://doi. org/10.1016/J.JACC.2020.05.001

5. Grillet F, Behr J, Calame P, Aubry S, Delabrousse E. Acute pulmonary embolism associated with COVID-19 pneumonia detected with pulmonary CT angiography. 101148/radiol2020201544. 2020;296(3):E186-E188. https://doi.org/10.1148/RADIOL.20202 01544

6. Varga Z, Flammer AJ, Steiger P et al (2020) Endothelial cell infection and endotheliitis in COVID-19. Lancet 395(10234):14171418. https://doi.org/10.1016/S0140-6736(20)30937-5

7. G C, D W, W G, et al. Clinical and immunological features of severe and moderate coronavirus disease 2019. J Clin Invest. 2020;130(5):2620-2629. https://doi.org/10.1172/JCI137244

8. Singhania N, Bansal S, Nimmatoori DP, Ejaz AA, McCullough PA, Singhania G (2020) Current overview on hypercoagulability in COVID-19. Am J Cardiovasc Drugs 20(5):1. https://doi.org/10. 1007/S40256-020-00431-Z

9. Abou-Ismail MY, Diamond A, Kapoor S, Arafah Y, Nayak L (2020) The hypercoagulable state in COVID-19: incidence, pathophysiology, and management. Thromb Res 194:101. https://doi. org/10.1016/J.THROMRES.2020.06.029

10. M O, HR B, D K, et al. Diagnosis, prevention, and treatment of thromboembolic complications in COVID-19: report of the National Institute for Public Health of the Netherlands. Radiology. 2020;297(1):E216-E222. https://doi.org/10.1148/RADIOL.20202 01629

11. S Z, Y W, T Z, L X. CT features of coronavirus disease 2019 (COVID-19) pneumonia in 62 patients in Wuhan, China. AJR Am J Roentgenol. 2020;214(6):1287-1294. https://doi.org/10.2214/ AJR.20.22975

12. Ng M-Y, Lee EYP, Yang J, et al. Imaging profile of the COVID19 infection: radiologic findings and literature review. 101148/ ryct2020200034. 2020;2(1). https://doi.org/10.1148/RYCT.20202 00034

13. Caruso D, Zerunian M, Polici M et al (2020) Chest CT features of COVID-19 in Rome, Italy. Radiology 296(2):E79-E85. https:// doi.org/10.1148/RADIOL.2020201237

14. Lang M, Som A, Carey D, et al. Pulmonary vascular manifestations of COVID-19 pneumonia. 101148/ryct2020200277. 2020;2(3). https://doi.org/10.1148/RYCT.2020200277

15. Poyiadji N, Cormier P, Patel PY, et al. Acute pulmonary embolism and COVID-19. 101148/radiol2020201955. 2020;297(3):E335-E338. https://doi.org/10.1148/RADIOL.20202 01955

16. C L, G I, L C, et al. Venous and arterial thromboembolic complications in COVID-19 patients admitted to an academic hospital in Milan, Italy. Thromb Res. 2020;191:9-14. https://doi.org/10. 1016/J.THROMRES.2020.04.024

17. Klok FA, Kruip MJHA, van der Meer NJM et al (2020) Incidence of thrombotic complications in critically ill ICU patients with COVID-19. Thromb Res 191:145. https://doi.org/10.1016/J. THROMRES.2020.04.013

18. D B, L B, L A, et al. Incidence of deep venous thrombosis in COVID-19 hospitalized patients during the first peak of the Italian outbreak. Phlebology. 2021;36(5):375-383. https://doi.org/ $10.1177 / 0268355520975592$

19. E L, A K, C C, D O, T H, S L. Arterial and venous thromboembolic complications of COVID-19 detected by CT angiogram and venous duplex ultrasound. Emerg Radiol. 2021;28(3):469-476. https://doi.org/10.1007/S10140-020-01884-0

20. A O, A P, S H, SI L. Multisystem assessment of the imaging manifestations of coagulopathy in hospitalized patients with coronavirus disease (COVID-19). AJR Am J Roentgenol. 2021;216(4):1088-1098. https://doi.org/10.2214/AJR.20.24132
21. C V, PA B, M DC, et al. Association between pulmonary embolism and COVID-19 severe pneumonia: experience from two centers in the core of the infection Italian peak. Eur J Radiol. 2021;137. https://doi.org/10.1016/J.EJRAD.2021.109613

22. Ooi MWX, Rajai A, Patel R, Gerova N, Godhamgaonkar V, Liong SY (2020) Pulmonary thromboembolic disease in COVID-19 patients on CT pulmonary angiography - prevalence, pattern of disease and relationship to D-dimer. Eur $\mathrm{J}$ Radiol 132:109336. https://doi.org/10.1016/J.EJRAD.2020. 109336

23. M M, RL Z, KW S, et al. VTE in ICU patients with COVID-19. Chest. 2020;158(5):2130-2135. https://doi.org/10.1016/J.CHEST. 2020.07.031

24. Cau R, Pacielli A, Fatemeh H et al (2021) Complications in COVID-19 patients: characteristics of pulmonary embolism. Clin Imaging 77:244. https://doi.org/10.1016/J.CLINIMAG.2021.05. 016

25. H A-S, RS KL, WH D, et al. COVID-19 and coagulation: bleeding and thrombotic manifestations of SARS-CoV-2 infection. Blood. 2020;136(4):489-500. https://doi.org/10.1182/BLOOD.20200 06520

26. Khurram R, Karia P, Naidu V, Quddus A, Woo WL, Davies N. Pulmonary artery pseudoaneurysm secondary to COVID-19 treated with endovascular embolisation. Eur J Radiol Open. 2021;8. https://doi.org/10.1016/J.EJRO.2021.100346

27. Savić D, Alsheikh TM, Alhaj AK et al (2020) Ruptured cerebral pseudoaneurysm in an adolescent as an early onset of COVID-19 infection: case report. Acta Neurochir (Wien) 162(11):1. https:// doi.org/10.1007/S00701-020-04510-7

28. Iba T, Levy JH, Warkentin TE, Thachil J, van der Poll T, Levi M (2019) Diagnosis and management of sepsis-induced coagulopathy and disseminated intravascular coagulation. J Thromb Haemost 17(11):1989-1994. https://doi.org/10.1111/JTH.14578

29. Le BA, Marteau V, Emmerich J, Zins M (2020) Concomitant acute aortic thrombosis and pulmonary embolism complicating COVID-19 pneumonia. Diagn Interv Imaging 101(5):321. https:// doi.org/10.1016/J.DIII.2020.04.003

30. Baltrūnas T, Račytė A, Pikturnaitė G, Skrebūnas A, Vaišnytė B. Aortic thrombosis after DVT and PE in a young COVID-19 patient. Ann Vasc Surg. Published online August 2021. https://doi. org/10.1016/J.AVSG.2021.07.005

31. Tinelli G, Sica S, Montanari F et al (2021) Spontaneous acute aortic thrombosis in SARS-CoV-2 infection. Ann Vasc Surg 75:136. https://doi.org/10.1016/J.AVSG.2021.04.010

32. Goldberg-Stein S, Fink A, Paroder V, Kobi M, Yee J, Chernyak V (2020) Abdominopelvic CT findings in patients with novel coronavirus disease 2019 (COVID-19). Abdom Radiol (New York) 45(9):1. https://doi.org/10.1007/S00261-020-02669-2

33. N H, PVA P, JAB A-F, et al. Abdominal gastrointestinal imaging findings on computed tomography in patients with COVID-19 and correlation with clinical outcomes. Eur J Radiol open. 2021;8. https://doi.org/10.1016/J.EJRO.2021.100326

34. HV V, A S, EK F, RS G. CT angiography of abdomen and pelvis in critically ill COVID-19 patients: imaging findings and correlation with the CT chest score. Abdom Radiol (New York). 2021;46(7):3490-3500. https://doi.org/10.1007/ S00261-021-03164-Y

35. Angileri SA, Petrillo M, Meglio L Di, et al. Adverse events in coronavirus disease patients management: a pictorial essay. J Clin Imaging Sci. 2020;10(1). https://doi.org/10.25259/JCIS_72_2020

36. Javid A, Kazemi R, Dehghani M, Samani HB (2021) Catastrophic retroperitoneal hemorrhage in COVID-19 patients under anticoagulant prophylaxis. Urol Case Reports 36:101568. https://doi. org/10.1016/J.EUCR.2021.101568

37. Bhayana R, Som A, Li MD, et al. Abdominal imaging findings in COVID-19: preliminary observations. 101148/radiol2020201908. 
2020;297(1):E207-E215. https://doi.org/10.1148/RADIOL.20202 01908

38. Chen J, Hang Y (2021) Characteristics, risk factors and outcomes of gastrointestinal hemorrhage in COVID-19 patients: a metaanalysis. Pakistan J Med Sci 37(5):1524. https://doi.org/10.12669/ PJMS.37.5.4351

39. Goyal H, Sachdeva S, Perisetti A, et al. Management of gastrointestinal bleeding during COVID-19: less is more! Eur J Gastroenterol Hepatol. Published online 2021:1230-1232. https://doi. org/10.1097/MEG.0000000000002224

40. Bargellini I, Cervelli R, Lunardi A et al (2020) Spontaneous bleedings in COVID-19 patients: an emerging complication. Cardiovasc Intervent Radiol 43(7):1. https://doi.org/10.1007/ S00270-020-02507-4

41. Abdelmohsen MA, Alkandari BM, Razek AAKA, Tobar AM, Gupta VK, Elsebaie N (2021) Abdominal computed tomography angiography and venography in evaluation of hemorrhagic and thrombotic lesions in hospitalized COVID-19 patients. Clin Imaging 79:12. https://doi.org/10.1016/J.CLINIMAG.2021.04.002

42. Nakamura H, Ouchi G, Miyagi K et al (2021) Case report: iliopsoas hematoma during the clinical course of severe COVID-19 in two male patients. Am J Trop Med Hyg 104(3):1018-1021. https://doi.org/10.4269/AJTMH.20-1507

43. Gawande RS, Vadvala HV, Shan A, Sheth S (2021) Ultrasound studies of COVID-19-positive patients and patient under investigation: pandemic experience of body imaging division at a tertiary medical center. Ultrasound Q 37(3):254-260. https://doi.org/10. 1097/RUQ.0000000000000571

44. Goodman LR, Stein PD, Matta F, et al. CT venography and compression sonography are diagnostically equivalent: data from PIOPED II. 102214/AJR072388. 2012;189(5):1071-1076. https:// doi.org/10.2214/AJR.07.2388

45. Chen B, Jiang C, Han B et al (2021) High prevalence of occult thrombosis in cases of mild/moderate COVID-19. Int J Infect Dis 104:77-82. https://doi.org/10.1016/J.IJID.2020.12.042

46. M O, FX D, AS S, A R, A K, B F. Peripheral arterial occlusion due to COVID-19: CT angiography findings of nine patients. Clin Imaging. 2021;73:43-47. https://doi.org/10.1016/J.CLINIMAG. 2020.11.023

47. IA G, K Y, MH S. Lower-extremity arterial thrombosis associated with COVID-19 is characterized by greater thrombus burden and increased rate of amputation and death. Radiology. 2020;297(2):E263-E269. https://doi.org/10.1148/RADIOL.20202 02348

48. Antonio G, Davide V, Marco G, et al. Retrospective survey from vascular access team Lombardy net in COVID-19 era. $J$ Vasc Access. Published online February 23, 2021. https://doi.org/10. $1177 / 1129729821997252$

49. Siguret V, Voicu S, Neuwirth M et al (2020) Are antiphospholipid antibodies associated with thrombotic complications in critically ill COVID-19 patients? Thromb Res 195:74-76. https://doi.org/ 10.1016/J.THROMRES.2020.07.016
50. Gupta S, Aly R, Gupta S, Singh B, Goyal A, Kalathil S. Case report: heparin-induced thrombocytopenia in a patient with COVID-19. F1000Research. 2020;9. https://doi.org/10.12688/ F1000RESEARCH.24974.2

51. Kubiak A, Chauhan N, Haque H, et al. Abstract No. 527 High incidence of large-bore temporary hemodialysis catheter thrombosis in patient with COVID-19-related kidney injury. $J$ Vasc Interv Radiol. 2021;32(5):S141. https://doi.org/10.1016/J.JVIR.2021.03. 336

52. Godon A, Durand Z, Agier L et al (2021) Comparison of fibrin monomers and D-dimers to predict thrombotic events in critically ill patients with COVID-19 pneumonia: a retrospective study. Thromb Res 205:8-10. https://doi.org/10.1016/J.THROMRES. 2021.06.009

53. Cattaneo M, Bertinato EM, Birocchi S et al (2020) Pulmonary embolism or pulmonary thrombosis in COVID-19? Is the recommendation to use high-dose heparin for thromboprophylaxis justified? Thromb Haemost 120(8):1230-1232. https://doi.org/10. 1055/S-0040-1712097/ID/JR200240-11

54. Thomas W, Varley J, Johnston A et al (2020) Thrombotic complications of patients admitted to intensive care with COVID-19 at a teaching hospital in the United Kingdom. Thromb Res 191:76-77. https://doi.org/10.1016/J.THROMRES.2020.04.028

55. Ferroli P, Villa C, Ciuffi A, Gubertini G, Broggi M (2020) Long lasting hypercoagulability after subclinical COVID-19. J Thromb Thrombolysis 50(4):822-824. https://doi.org/10.1007/S11239020-02215-3/FIGURES/1

56. Whitworth H, Sartain SE, Kumar R et al (2021) Rate of thrombosis in children and adolescents hospitalized with COVID-19 or MIS-C. Blood 138(2):190-198. https://doi.org/10.1182/BLOOD. 2020010218

57. Maurer LR, Luckhurst CM, Hamidi A et al (2020) A low dose heparinized saline protocol is associated with improved duration of arterial line patency in critically ill COVID-19 patients. J Crit Care 60:253-259. https://doi.org/10.1016/J.JCRC.2020.08.025

58. Al-Thani H, Hussein A, Sadek A, Barah A, El-Menyar A (2021) Balloon-assisted percutaneous thrombin injection for treatment of iatrogenic left subclavian artery pseudoaneurysm in a critically ill COVID-19 patient. Case Rep Vasc Med 2021:1-6. https://doi. org/10.1155/2021/4245484

59. Rotzinger DC, Beigelman-Aubry C, von Garnier C, Qanadli SD (2020) Pulmonary embolism in patients with COVID-19: time to change the paradigm of computed tomography. Thromb Res 190:58. https://doi.org/10.1016/J.THROMRES.2020.04.011

Publisher's note Springer Nature remains neutral with regard to jurisdictional claims in published maps and institutional affiliations. 